\title{
KRZYSZTOF MOSZYŃSKI
}

\section{Approximation spectral d'un opérateur borné et normal à l'aide de ses fonctions de la densité spectrale}

RAIRO - Analyse numérique, tome 17, nº 1 (1983), p. 93-109.

<http://www.numdam.org/item?id=M2AN_1983_17_1_93_0>

(C) AFCET, 1983, tous droits réservés.

L'accès aux archives de la revue «RAIRO - Analyse numérique » implique l'accord avec les conditions générales d'utilisation (http://www.numdam.org/ legal.php). Toute utilisation commerciale ou impression systématique est constitutive d'une infraction pénale. Toute copie ou impression de ce fichier doit contenir la présente mention de copyright.

\section{Numdam}




\title{
APPROXIMATION SPECTRALE D'UN OPERATEUR BORNE ET NORMAL A L'AIDE DE SES FONCTIONS DE LA DENSITÉ SPECTRALE $\left({ }^{*}\right)$
}

\author{
par Krzysztof MoszYŃsKi ( $\left.{ }^{1}\right)$
}

Communiqué par P. G. Ciarlet

Résumé. - On présente une méthode d'approximation des fonctions de la densité spectrale $\phi_{x}(\lambda)=\|E(\lambda) x\|^{2} d^{\prime}$ 'un opérateur borné et normal A dont la familte spectrate est $E(\lambda)$.

La méthode est basée sur la construction de certaines formules de quadrature approchée en deux dimensions, à coefficients positifs. Les fonctions $\phi_{x}$ sont utilisées pour l'approximation du spectre $\sigma(A)$.

Summary. - This paper concerns a method of approximation of the spectral density functions $\phi_{x}(\lambda)=\|E(\lambda) x\|^{2}$ of a bounded, normal operator $A$ with the spectral family $E(\lambda)$. The method is based on a construction of certain approximate quadrature formulas in two dimensions, with positive coefficients. The functions $\phi_{x}$ are used to approximate the spectrum $\sigma(A)$.

\section{INTRODUCTION}

Dans [4] on a présenté une méthode d'approximation spectrale applicable aussi dans le cas du spectre continu d'un opérateur autoadjoint. Ce papier généralise certains résultats de [4].

Soit $H$ un espace de Hilbert complexe, muni du produit scalaire ()$_{H}$ et $A=\int_{\Omega} \lambda d E(\lambda): H \rightarrow H$ un opérateur borné et normal quelconque. Ici $\Omega$ est un rectangle borné et fermé dans le plan complexe $\mathbb{C}$ :

$$
\Omega=\left\{\lambda=u+i v=(u, v) \in \mathbb{C} \mid a_{1} \leqslant u \leqslant a_{2}, b_{1} \leqslant v \leqslant b_{2}\right\},
$$

contenant le spectre $\sigma(A)$ de $A$. On dénotera :

$$
\rho(A)=\mathbb{C} \backslash \sigma(A) .
$$

(*) Reçu en décembre 1981.

(') Instytut Matematyczny PAN ul. Sniadeckich 8, 00-950 Warszawa, Boîte postale 137, Pologne.

R.A.I.R.O. Analyse numérique/Numerical Analysis, 0399-0516/1983/93/\$ 6.00

(C) AFCET Bordas-Dunod 
Tout rectangle borné et fermé $Q \subset \mathbb{C}$, dont les côtés sont parallèles aux axes des coordonnées de $\mathbb{C}$ est appelé ici un rectangle normal. On fixe une fois pour toutes le mode de numération des sommets $q_{1}, q_{2}, q_{3}, q_{4}$ d'un tel rectangle :

$$
q_{1}=(\alpha, \gamma), \quad q_{2}=(\beta, \gamma), \quad q_{3}=(\beta, \delta), \quad q_{4}=(\alpha, \delta)
$$

avec $\alpha<\beta$ et $\gamma<\delta$.

L'ensemble $L B(Q)=\left[q_{1}, q_{2}\right] \cup\left[q_{1}, q_{4}\right]$ est appelé ici la frontière inférieure (lower boundary) de $Q$ [8].

La famille spectrale $E$ de l'opérateur $A$ se décompose d'une telle manière que $E(\lambda)=E_{1}(u) E_{2}(v)=E_{2}(v) E_{1}(u)$ où $E_{1}$ est la famille de la partie réelle $R=\frac{A+A^{*}}{2}$ de $A$ et $E_{2}$ celle de sa partie imaginaire $S=\frac{A-A^{*}}{2 i}$. Il nous sera convenable de modifier éventuellement la définition classique (voir [6]) en posant $E_{1}\left(a_{1}\right)=0$ et $E_{2}\left(b_{1}\right)=0$. Comme, d'une manière évidente $E_{1}$ et $E_{2}$ se prolongent en dehors de $\left[a_{1}, a_{2}\right]$ et $\left[b_{1}, b_{2}\right]$ respectivement, la famille spectrale $E$ de $\boldsymbol{A}$ devient une fonction définie sur $\mathbb{C}$ entier, dont les valeurs sont des projections orthogonales dans $H$. D'ailleurs $E$ est continue (fort) de droite par rapport à chacune de ses deux variables $u$ et $v$ dans Int $(\Omega)$ et s'annule sur $L B(\Omega)$.

Pour tout $x \in H$ fixé, on définit la fonction de la densité spectrale de l'opérateur $A \phi_{x}: \mathbb{C} \rightarrow \mathbb{R}:$

$$
\phi_{x}(\lambda)=(E(\lambda) x, x)_{H}=\|E(\lambda) x\|_{H}^{2} \geqslant 0 .
$$

Soit $Q$ un rectangle normal avec les sommets $q_{1}, q_{2}, q_{3}, q_{4}$ et $f$ une fonction définie sur $\mathbb{C}$. Mettons

$$
\Delta_{Q} f=f\left(q_{1}\right)+f\left(q_{3}\right)-f\left(q_{2}\right)-f\left(q_{4}\right) .
$$

On appelle $\Delta_{Q} f$ la différence de $f$ sur $Q$. On dit que la fonction réelle $f$ est entièrement croissante, si pour tout rectangle normal $Q, \Delta_{Q} f \geqslant 0$ et si $f$ est croissante par rapport à $u$ et $v$ respectivement sur les deux segments $\left[q_{1}, q_{2}\right]$ et $\left[q_{1}, q_{4}\right]$ de $L B(Q)$. On voit facilement que $\phi_{x}$ est entièrement croissante.

Pour $\lambda=(u, v) \in \mathbb{C}$ et $h>0$, définissons le rectangle normal $Q(\lambda, h)$ par ses sommets $q_{1}, q_{2}, q_{3}, q_{4}$ :

$$
\begin{aligned}
q_{1}=(u-h, v-h), & q_{2}=(u+h, v-h), \quad q_{3}=(u+h, v+h), \\
q_{4} & =(u-h, v+h) .
\end{aligned}
$$

Le théorème 1.1 suit directement du matériel classique (pour la démonstration détaillée, voir [5]). 
THÉORÈME 1.1 : (i) Pour tout rectangle normal $Q \subset \mathbb{C}$ :

- s'il existe $x \in H$ tel que $\Delta_{Q} \phi_{x}>0$, alors $Q \cap \sigma(A) \neq \varnothing$;

- si $\sigma(A) \cap \operatorname{Int}(Q) \neq \varnothing$, alors il existe $x \in H$ tel que $\Delta_{Q} \phi_{x}>0$;

(ii) $\lambda$ est une valeur propre de A si et seulement si on peut trouver un tel élément $x \in H$ que

$$
\Delta_{Q}(\lambda, h) \phi_{x} \rightarrow v>0 \text { quand } h \rightarrow 0 .
$$

D'ailleurs $v=\|\Delta(\lambda) x\|_{H}^{2}$ où $\Delta(\lambda)=\lim _{n \rightarrow 0} \Delta_{Q(\lambda, h)} E$ (fort);

(iii) $\lambda$ est dans le spectre continu de l'opérateur $A$ si et seulement si pour tout $h>0$ on peut trouver un $x_{h} \in H$, tel que $\Delta_{Q(\lambda, h)} \phi_{x_{h}}>0$, tandis que $\Delta_{Q(\lambda, h)} \phi_{x} \rightarrow 0$ quand $h \rightarrow 0$, pour tout $x \in H$. ||

Soit $\Omega$ un rectangle normal tel que $\sigma(A) \subset \Omega$. Le théorème 1.1 permet de classifier les points de $\Omega$ de la manière suivante. Divisons le rectangle $\Omega$ à l'aide d'un réseau des axes parallèles aux côtés de $\Omega$ pour obtenir sa décomposition en un nombre fini de rectangles normaux, plus petits, de diamètre $\leqslant h$. Soit $Q$ un tel rectangle. Si $\Delta_{Q} \phi_{x}>0$ pour un $x \in H$, alors on a $Q \cap \sigma(A) \neq \varnothing$. Ainsi on peut se servir de la fonction $\phi_{x}$ afin d'approximer une partie du spectre $\sigma(A)$ avec la précision définie par $h$. Mais en général on ne connaît pas de fonctions $\phi_{x}$. Donc l'approximation des fonctions $\phi_{x}$ paraît être un problème intéressant du point de vue pratique. Pour avoir le spectre entier $\sigma(A)$, il faut qu'on puisse approximer $\phi_{x}$ pour tout $x \in H$, ce qui, bien sûr, n'est pas exécutable. On peut se servir à cet effet d'un réseau fini de points de $H$, au moins dans le cas où $H$ est séparable. Par exemple des bases des sous-espaces de dimension finie, approximant $H$ peuvent être appliquées (voir par ex. [1]). Certaines idées concernant ce genre d'approximation sont présentées dans [4]. Les points (i) et (ii) du théorème 1.1 peuvent être utiles pour la classification du spectre même (valeurs propres, spectre continu).

Conclusion : L'approximation des fonctions de la densité spectrale $\phi_{x}$ permet d'approximer le spectre $\sigma(A)$ et éventuellement classifier les points du spectre.

On présente ici une méthode d'approximation effective des fonctions $\phi_{x}$. On y trouvera également deux propositions d'algorithmes.

\section{QUELQUES LEMMES SUR APPROXIMATION}

On se donne une fonction $\phi: \Omega \rightarrow \mathbb{R}$ définie sur un rectangle normal $\Omega$, telle que :

- $\phi$ est entièrement croissante sur $\Omega$, 
- $\phi$ est normalisée [8] ; c'est-à-dire que :

a) $\phi$ est continue de droite dans Int $(\Omega)$ par rapport à chacune de ses variables;

b) $\phi$ s'annule sur $L B(\Omega)$.

Exemple : fonction de la densité spectrale $\phi_{x}$.

Pour $\lambda, \mu \in \mathbb{C}, \lambda=(u, v), \mu=(r, s)$, soit $H(\lambda, \mu)=H(u, r) . H(v, s)$ avec

$$
H(x, y)=\left\{\begin{array}{lll}
0 & \text { pour } x<y \\
1 & \text { pour } x \geqslant y
\end{array},\right.
$$

pour tous $x, y$ réels. Mettons pour tout $d$ naturel :

$$
\phi_{d}(\lambda)=\sum_{j=1}^{N(d)} B_{j}^{d} H\left(\lambda, \mu_{j}^{d}\right)
$$

où $N(d)$ naturel, $B_{j}^{d} \geqslant 0, \mu_{j}^{d} \in \Omega \backslash L B(\Omega), j=1,2, \ldots, N(d)$. On vérifie facilement que :

(i) $\phi_{d}$ est entièrement croissante et normalisée;

(ii) la variation totale $\bigvee_{\Omega} \phi_{d}$ de $\phi_{d}$ sur $\Omega$ satisfait $\bigvee_{\Omega} \phi_{d}=\int_{\Omega} d \phi_{d}(\lambda)=\sum_{j=1}^{N(d)} B_{j}^{d}$,

(iii) $\int_{\Omega} f(\lambda) d \phi_{d}(\lambda)=\sum_{j=1}^{N(d)} B_{j}^{d} f\left(\mu_{j}^{d}\right)$ pour toute fonction continue $f \in C(\Omega)$.

La condition suivante $(Q)$ nous sera utile.

Pour toute fonction réelle continue $f$

$$
\text { (Q) } \int_{\Omega} f(\lambda) d \phi_{d}(\lambda) \rightarrow \int_{\Omega} f(\lambda) d \phi(\lambda)
$$

quand $d \rightarrow \infty$.

Rappelons encore ici la formule d'intégration par parts [8] : Soit $Q$ un rectangle normal, $q_{1}, q_{2}, q_{3}, q_{4}$ ses sommets, $\partial Q$ sa frontière, $f$ continue sur $Q$. Si $\int_{Q} f d \phi$ et $\int_{\partial Q} f d \phi$ existent, alors $\int_{Q} \phi d f$ et $\int_{\partial Q} \phi d f$ existent, et :

$$
\begin{aligned}
\int_{Q} f d \phi=\Delta_{Q}(f \phi)+\int_{q_{1} q_{2}} \phi d f+\int_{q_{1} q_{4}} \phi d f- & \\
& -\int_{q_{2} q_{3}} \phi d f-\int_{q_{4} q_{3}} \phi d f+\int_{Q} \phi d f .
\end{aligned}
$$


Lemme 2.1 : Soit $Q \subset \Omega$ un rectangle normal dont les sommets sont $q_{1}, q_{2}$, $q_{3}, q_{4}$. Si la condition $(Q)$ est satisfaite, alors

$$
\int_{Q} \phi_{d}(r, s) d r d s \rightarrow \int_{Q} \phi(r, s) d r d s \text { quand } d \rightarrow \infty
$$

Démonstration (l'idée de la démonstration : voir [6]) : Pour tout $\lambda=(u, v) \in \Omega$, soit :

$$
\begin{aligned}
& \Omega(\lambda)=\{(r, s) \in \Omega \mid r \leqslant u \text { et } s \leqslant v\}, \\
& \psi(\lambda)=\int_{\Omega(\lambda)} \phi(r, s) d r d s, \\
& \psi_{d}(\lambda)=\int_{\Omega(\lambda)} \phi_{d}(r, s) d r d s .
\end{aligned}
$$

Mettons pour $\lambda \in \Omega$ fixé et $\mu=(\xi, \zeta) \in \mathbb{C}$

$$
f_{\lambda}(\mu)=f_{\lambda}(\xi, \zeta)=\left\{\begin{array}{cl}
(u-\xi)(v-\zeta) & \text { pour }(\xi, \zeta) \in \Omega(\lambda) \\
0 & \text { ailleurs }
\end{array}\right.
$$

Comme $f_{\lambda}$ est continue par rapport à $\xi$ et $\zeta$, la condition $(Q)$ entraîne :

$$
F_{d}\left(f_{\lambda}\right)=\int_{\Omega} f_{\lambda}(\mu) d \phi_{d}(\mu) \rightarrow F\left(f_{\lambda}\right)=\int_{\Omega} f_{\lambda}(\mu) d \phi(\mu)
$$

pour tout $\lambda \in \Omega$, quand $d \rightarrow \infty$.

L'intégration par parts entraîne :

$$
F_{d}\left(f_{\lambda}\right)=\int_{\Omega} f_{\lambda}(\mu) d \phi_{d}(\mu)=\int_{\Omega(\lambda)} f_{\lambda}(\mu) d \phi_{d}(\mu)=\int_{\Omega(\lambda)} \phi_{d}(\xi \zeta) d \xi d \zeta=\psi_{d}(\lambda)
$$

et de la manière analogue $F\left(f_{\lambda}\right)=\psi(\lambda)$.

Donc $\psi_{d}(\lambda) \rightarrow \psi(\lambda)$ quand $d \rightarrow \infty$ pour tout $\lambda \in \Omega$.

Pour avoir (2.2) il suffit d'observer que

$$
\begin{aligned}
& \int_{Q} \phi(r, s) d r d s=\Delta_{Q} \psi \\
& \int_{Q} \phi_{d}(r, s) d r d s=\Delta_{Q} \psi_{d \cdot} \quad \|
\end{aligned}
$$


LeMME 2.2 : (l'idée de la démonstration : voir [6]) : Si la condition $(Q)$ est satisfaite, alors pour tout $\lambda=(u, v) \in \ln t(\Omega)$ on $a$ :

$$
\begin{aligned}
& \phi(u-0, v-0)-\phi(u, v) \leqslant \lim _{d \rightarrow \infty}\left[\phi(\lambda)-\phi_{d}(\lambda)\right] \leqslant \\
& \leqslant \varlimsup_{d \rightarrow \infty}\left[\phi(\lambda)-\phi_{d}(\lambda)\right] \leqslant \phi(u, v)-\phi(u-0, v-0) .
\end{aligned}
$$

Démonstration : Soit $\lambda=(u, v) \in \operatorname{Int}(\Omega)$. Comme $\phi$ et $\phi_{d}$ sont normalisées et entièrement croissantes, le calcul facile nous amène à la conclusion que

$$
\begin{aligned}
& \frac{1}{k h}\left[\int_{u-h}^{u} \int_{v-k}^{v} \phi(r, s) d r d s-\int_{u}^{u+h} \int_{v}^{v+k} \phi_{d}(r, s) d r d s\right] \leqslant \\
& \quad \leqslant \phi(\lambda)-\phi_{d}(\lambda) \leqslant \frac{1}{k h}\left[\int_{u}^{u+h} \int_{v}^{v+k} \phi(r s) d r d s-\int_{u-h}^{u} \int_{v-k}^{v} \phi_{d}(r s) d r d s\right] .
\end{aligned}
$$

Passons d'abord à la limite avec $d \rightarrow \infty$, et puis avec $(k, h) \rightarrow(0,0)$. Il en résulte l'inégalité voulue. I||

COROllaire : Si $\phi$ est continue au point $\lambda$, alors

$$
\phi_{d}(\lambda) \rightarrow \phi(\lambda) \text { quand } d \rightarrow \infty .
$$

LEMME 2.3 : Si la condition $(Q)$ est satisfaite, alors pour tout $\rho \in L^{2}(\Omega)$

$$
\int_{\Omega} \rho(u, v) \phi_{d}(u, v) d u d v \rightarrow \int_{\Omega} \rho(u, v) \phi(u, v) d u d v \quad \text { quand } d \rightarrow \infty .
$$

Démonstration : Mettons $f(u, v)=\int_{a_{2}}^{u} \int_{b_{2}}^{v} \rho(r, s) d r d s$, où $\left(a_{1}, b_{1}\right)\left(a_{2}, b_{1}\right)$ $\left(a_{2}, b_{2}\right),\left(a_{1}, b_{2}\right)$ sont les sommets du rectangle normal $\Omega$. Intégration par parts appliquée à $\int_{\Omega} f(\lambda) d \phi(\lambda)$ et $\int_{\Omega} f(\lambda) d \phi_{d}(\lambda)$, la condition de normalité de $\Omega$ et la condition $(Q)$ entraînent que

$$
\begin{aligned}
\int_{\Omega} f(\lambda) d \phi_{d}(\lambda)=\int_{\Omega} \rho(r, s) \phi_{d}(r, s) d r & d s \\
& \rightarrow \int_{\Omega} f(\lambda) d \phi(\lambda)=\int_{\Omega} \rho(r, s) \phi(r s) d r d s
\end{aligned}
$$

quand $d \rightarrow \infty$. $\|$ 
LEMME 2.4 : (l'idée de la démonstration : voir [3]) : Soit $S \subset \mathbb{C}$ l'union des rectangles normaux et des segments fermés, bornés, parallèles aux axes des coordonnées de $\mathbb{C}$.

Soit $\left\{\phi_{d}\right\}$ la suite des fonctions $\phi_{d}: \mathbb{C} \rightarrow \mathbb{R}$, croissantes par rapport à chacune de ses deux variables, telles que $\phi_{d}(\lambda) \rightarrow \phi(\lambda)$ quand $d \rightarrow \infty$, pour tout $\lambda \in S$. Supposons que $\phi: \mathbb{C} \rightarrow \mathbb{R}$ est continue pour tout point $\lambda \in S$.

Dans ce cas $\phi_{d}$ converge uniformément vers $\phi$ sur $S$.

Démonstration : Soit d'abord $S$, un rectangle normal. Étant donné $\varepsilon>0$, divisons $S$ à l'aide d'un réseau des droites parallèles aux côtés de $S$, pour obtenir un système des rectangles $Q_{1}, Q_{2}, \ldots, Q_{N(\varepsilon)}$, dont les sommets sont $p_{1}^{j}, p_{2}^{j}, p_{3}^{j}$ et $p_{4}^{j}, j=1,2, \ldots, N(\varepsilon)$ respectivement.

On suppose, que la condition

$$
\left|\phi\left(p_{k}^{j}\right)-\phi\left(p_{l}^{j}\right)\right|<\varepsilon k, l=1,2,3,4 \text { et } j=1,2, \ldots, N(\varepsilon)
$$

est satisfaite ( $\phi$ est uniformément continue sur le compact $S$ ).

Pour tout $x \in S$ trouvons un rectangle $Q_{j}$ tel que $x \in Q_{j}$.

On a

$$
\begin{aligned}
\phi(x)- & \phi_{d}(x) \leqslant \phi(x)-\phi_{d}\left(p_{1}^{j}\right) \leqslant \\
& \leqslant\left|\phi(x)-\phi\left(p_{1}^{j}\right)\right|+\left|\phi\left(p_{1}^{j}\right)-\phi_{d}\left(p_{1}^{j}\right)\right| \leqslant \varepsilon+\left|\phi\left(p_{1}^{j}\right)-\phi_{d}\left(p_{1}^{j}\right)\right|
\end{aligned}
$$

et

$$
\begin{aligned}
& \phi(x)-\phi_{d}(x) \geqslant \phi(x)-\phi_{d}\left(p_{3}^{j}\right) \geqslant \\
& \quad \geqslant-\left|\phi(x)-\phi\left(p_{3}^{j}\right)\right|-\left|\phi\left(p_{3}^{j}\right)-\phi_{d}\left(p_{3}^{j}\right)\right| \geqslant-\varepsilon-\left|\phi\left(p_{3}^{j}\right)-\phi_{d}\left(p_{3}^{j}\right)\right|
\end{aligned}
$$

donc

$$
-\varepsilon-\left|\phi\left(p_{3}^{j}\right)-\phi_{d}\left(p_{3}^{j}\right)\right| \leqslant \phi(x)-\phi_{d}(x) \leqslant \varepsilon+\left|\phi\left(p_{1}^{j}\right)-\phi_{d}\left(p_{1}^{j}\right)\right| .
$$

Comme $\phi_{d}(\lambda) \rightarrow \phi(\lambda)$ quand $d \rightarrow \infty$ et $\lambda \in S$, on peut trouver $M_{\varepsilon}$, tel que

$$
\begin{aligned}
& \left|\phi\left(p_{1}^{j}\right)-\phi_{d}\left(p_{1}^{j}\right)\right|<\varepsilon \\
& \left|\phi\left(p_{3}^{j}\right)-\phi_{d}\left(p_{3}^{j}\right)\right|<\varepsilon
\end{aligned}
$$

pour $d>M_{\varepsilon}$ et $j=1,2, \ldots, N(\varepsilon)$; c'est-à-dire que

$$
-2 \varepsilon \leqslant \phi(x)-\phi_{d}(x) \leqslant 2 \varepsilon
$$

si $d>M_{\varepsilon}$. Le raisonnement pareil s'applique dans le cas de segments, et en conséquence dans le cas de l'union finie des segments et rectangles. ||| 
Corollaire: Supposons que $\phi_{d}(\lambda) \geqslant 0$ pour tout det $\lambda=(u, v) \in \Omega$. Mettons

$$
\psi(\lambda)=\int_{\Omega(\lambda)} \phi(r, s) d r d s, \quad \psi_{d}(\lambda)=\int_{\Omega(\lambda)} \phi_{d}(r, s) d r d s .
$$

Les fonctions $\psi(\lambda)$ et $\psi_{d}(\lambda)$ sont continues et croissantes par rapport à chacune de ses variables. Il suit du lemme 2.1, que $\psi_{d}(\lambda) \rightarrow \psi(\lambda)$ quand $d \rightarrow \infty$ pour tout $\lambda \in \Omega$. Donc

$$
\psi_{d}(\lambda) \rightarrow \psi(\lambda) \text { quand } d \rightarrow \infty
$$

uniformément dans $\Omega$. $\quad \|$

\section{ALGORITHMES, CONVERGENCE}

On présente ici deux algorithmes pour l'approximation de la fonction de la densité spectrale $\phi_{x}$ de l'opérateur $A$.

Dans les deux cas on construit une suite des fonctions en escaliers $\left\{\phi_{x d}\right\}$ de la forme (2.1), entièrement croissantes et normalisées, qui approchent $\phi_{x}$ lorsque $d \rightarrow \infty$. Dans les deux cas l'algorithme est basé sur la construction d'une quadrature approchée sur un rectangle normal $\Omega$ contenant le spectre $\sigma(A)$, dont les coefficients sont non négatifs. Il faut souligner ici que cette dernière condition est d'une grande importance (voir la section précédente).

Algorithme 1 : Soit :

- $d$ un nombre naturel. Mettons $N(d)=(d+1)(d+2) / 2$;

$-\varphi_{1}, \varphi_{2}, \ldots, \varphi_{N(d)}$ une base quelconque de l'espace des polynômes réels $d u$ degré $\leqslant d$, en deux variables réelles;

$-x \in H$ un élément fixé.

a) Calculer les constantes $c_{k}, k=1,2, \ldots, N(d)$

$$
c_{k}=\left(\phi_{k}(R, S) x ; x\right)_{H}
$$

où $R$ et $S$ sont respectivement la partie réelle et la partie imaginaire de $A$. Observons que les coefficients $c_{k}$ peuvent être calculés effectivement (au moins approximativement) dans plusieurs cas intéressants.

b) Résoudre le problème suivant : Trouver

tels que

$$
\mu_{j}^{d} \in \Omega \backslash L B(\Omega) \quad \text { et } \quad B_{j}^{d} \geqslant 0, j=1,2, \ldots, N(d)
$$

$$
\sum_{j=1}^{N(d)} \varphi_{k}\left(\mu_{j}^{d}\right) B_{j}^{d}=c_{k}, \quad k=1,2, \ldots, N(d) .
$$


Mettre

$$
\phi_{x d}(\lambda)=\sum_{j=1}^{N(d)} B_{j}^{d} H\left(\lambda, \mu_{j}^{d}\right), \quad \lambda \in \Omega .
$$

Commentaire : Observons que dans le point $b$ ) il faut résoudre un système de $N(d)$ équations non linéaires avec $2 N(d)$ inconnues $B_{1}^{d}, \ldots, B_{N(d)}^{d}$ et $\mu_{1}^{d}, \ldots, \mu_{N(d)}^{d}$, satisfaisant aux conditions additionnelles

$$
\mu_{j}^{d} \in \Omega \backslash L B(\Omega) \quad \text { et } \quad B_{j}^{d} \geqslant 0, j=1,2, \ldots, N(d) .
$$

En appliquant le théorème spectral, on voit que

$$
c_{k}=\left(\varphi_{k}(R, S) x, x\right)_{H}=\left(\int_{\Omega} \varphi_{k}(\lambda) d E(\lambda) x, x\right)_{H}=\int_{\Omega} \varphi_{k}(\lambda) d \phi_{x}(\lambda),
$$

donc l'équation (3.1) s'écrit comme

$$
\sum_{j=1}^{N(d)} \varphi_{k}\left(\mu_{j}^{d}\right) B_{j}^{d}=\int_{\Omega} \varphi_{k}(\lambda) d \phi_{x}(\lambda) \quad k=1,2, \ldots, N(d) .
$$

Ainsi dans le point $b$ ) de l'algorithme, on cherche une formule de quadrature avec des coefficients positifs, qui soit exacte pour tous les polynômes du degré $\leqslant d$. On sait qu'une telle formule existe toujours (voir [9]). Il faut noter ici, qu'en élargissant éventuellement le rectangle $\Omega$, on s'assure que les nouds $\mu_{j}^{d} j=1,2, \ldots, N(d)$ se trouveront en dehors de $L B(\Omega)$.

Il reste le problème toujours ouvert d'approximation numérique de la solution de $b$ ).

THÉORÈME $3.1:$ Si $\phi_{x}(\lambda)=\|E(\lambda) x\|_{H}^{2}$ et $\phi_{x d}$ sont définies par l'algorithme 1 , alors la condition $(Q)$ est satisfaite. Donc dans ce cas :

(i) $\int_{\Omega} \rho(u, v) \phi_{x d}(u, v) d u d v \rightarrow \int_{\Omega} \rho(u, v) \phi_{x}(u, v) d u d v$ quand $d \rightarrow \infty$

(ii) $\phi_{x}(u-0, v-0)-\phi_{x}(u, v) \leqslant$

$$
\begin{aligned}
\leqslant \lim _{d \rightarrow \infty}\left[\phi_{x}(\lambda)-\phi_{x d}(\lambda)\right] & \leqslant \varlimsup_{d \rightarrow \infty}\left[\phi_{x}(\lambda)-\phi_{x d}(\lambda)\right] \\
& \leqslant \phi_{x}(u, v)-\phi_{x}(u-0, v-0)
\end{aligned}
$$

pour tout $\lambda=(u, v) \in \Omega$ et $\rho \in L^{2}(\Omega)$.

En plus, si $\phi_{x}$ est continue au point $\lambda \in \Omega$, alors

$$
\phi_{x d}(\lambda) \rightarrow \phi_{x}(\lambda) \text { quand } d \rightarrow \infty .
$$


Cette convergence est uniforme dans tout ensemble $S \subset \Omega$ qui est l'union finie des rectangles normaux et des segments bornés et fermés, parallèles aux côtés de $\Omega$, pourvu que $\phi_{x}$ soit continue à tout point de $S$.

Démonstration : Observons qu'il suffit de vérifier que la condition $(Q)$ est satisfaite. Les points (i) et (ii) suivent directement des lemmes 2.2 et 2.3.

Soit $Q_{m}$ un polynôme quelconque, en deux variables du degré $m \leqslant d$. Les formules (3.1), (3.2) et (3.3) impliquent

$$
\sum_{j=1}^{N(d)} B_{j}^{d} Q_{m}\left(\mu_{j}^{d}\right)=\int_{\Omega} Q_{m}(\lambda) d \phi_{x d}(\lambda)=\int_{\Omega} Q_{m}(\lambda) d \phi_{x}(\lambda)
$$

donc dans le cas particulier $Q_{m}(\lambda) \equiv 1$ on a

$$
\sum_{j=1}^{N(d)} B_{j}^{d}=\int_{\Omega} d \phi_{x d}(\lambda)=\int_{\Omega} d \phi_{x}(\lambda)=\|x\|_{H}^{2} .
$$

Soit $f$ une fonction continue, $f \in C_{R}(\Omega)$. On dénotera la norme " sup » dans l'espace des fonctions continues réelles $C_{R}(\Omega)$ par $\|\cdot\|_{\infty}$, Étant donné $\varepsilon>0$, on peut trouver un polynôme $Q_{m_{\varepsilon}}$ de degré $m_{\varepsilon}$ tel que $\left\|f-Q_{m_{\varepsilon}}\right\|_{\infty}<\varepsilon$. Nous avons

$$
\begin{aligned}
& \int_{\Omega} f(\lambda) d \phi_{x}(\lambda)-\int_{\Omega} f(\lambda) d \phi_{x d}(\lambda)= \\
&=\int_{\Omega}\left[f(\lambda)-Q_{m_{e}}(\lambda)\right] d \phi_{x}(\lambda)-\int_{\Omega}\left[f(\lambda)-Q_{m_{\varepsilon}}(\lambda)\right] d \phi_{x d}(\lambda)+ \\
&+\left[\int_{\Omega} Q_{m_{\varepsilon}}(\lambda) d \phi_{x}(\lambda)-\int_{\Omega} Q_{m_{2}}(\lambda) d \phi_{x d}(\lambda)\right] .
\end{aligned}
$$

Si $d \geqslant m_{\varepsilon}$, le troisième terme s'annule, donc (voir (3.4) et (3.5)) :

$$
\begin{aligned}
& \left|\int_{\Omega} f(\lambda) d \phi_{x}(\lambda)-\int_{\Omega} f(\lambda) d \phi_{x}(\lambda)\right| \leqslant \\
& \quad \leqslant\left\|f-Q_{m_{\varepsilon}}\right\|_{\infty}\left[\int_{\Omega} d \phi_{x}(\lambda)+\int_{\Omega} d \phi_{x d}(\lambda)\right] \leqslant 2 \varepsilon\|x\|_{H}^{2} .
\end{aligned}
$$

Cela prouve la condition $(Q)$. III

Algorithme 2 : Dans l'espace $C_{k}(\Omega)$ des fonctions réelles continues sur $\Omega$ introduisons le produit scalaire quelconque, réel ()$_{0}$, et la norme correspon- 
dante $\|\cdot\|_{0}$, tels que $\|f\|_{0} \leqslant \kappa\|f\|_{\infty}$ pour toute $f \in C_{R}(\Omega)$, où $\kappa$ est une constante.

Soit $\varphi_{1}, \varphi_{2}, \ldots, \varphi_{N(d)}, N(d)=(d+1)(d+2) / 2$, une base quelconque des polynômes en deux variables du degré $\leqslant d$, orthonormale par rapport au produit scalaire ()$_{0}$.

Fixons un élément $x \in H$.

a) Choisir une suite des nombres positifs $\varepsilon_{1}, \varepsilon_{2}, \ldots$, telle que $\varepsilon_{d} \rightarrow 0$ quand $d \rightarrow \infty$.

b) A l'aide d'un réseau des axes parallèles aux côtés de $\Omega$, diviser le rectangle $\Omega$ en $M(d)$ rectangles plus petits $Q_{1}^{d}, Q_{2}^{d}, \ldots, Q_{M(d)}^{d}$, d'une telle manière que le diamètre maximal de $Q_{j}^{\text {d }}$ converge à zéro, quand $M(d) \rightarrow \infty$. Choisir $\mu_{j}^{d} \in Q_{j}^{d}, j=1,2, \ldots, M(d)$ d'une manière arbitraire.

c) Calculer $c_{k}=\left(\varphi_{k}(R, S) x, x\right)_{H}, k=1,2, \ldots, N(d)$ où $A=R+i S$.

d) Pour $d$ fixé trouver :

- le nombre $M(d)$,

- les coefficients $B_{j}^{d} \geqslant 0, j=1,2, \ldots, M(d)$, tels que

$$
\min _{B_{j}^{d} \geqslant 0} \sum_{k=1}^{N(d)}\left[\sum_{j=1}^{M(d)} B_{j}^{d} \varphi_{k}\left(\mu_{j}^{d}\right)-c_{k}\right]^{2} \leqslant \varepsilon_{d} .
$$

e) Mettre

$$
\phi_{x d}(\lambda)=\sum_{j=1}^{M(d)} B_{j}^{d} H\left(\lambda, \mu_{j}^{d}\right) .
$$

Commentaire : Il y a diverses possibilités du choix d'un système orthonormal $\varphi_{1}, \varphi_{2}, \ldots, \varphi_{N(d)}$. Par exemple les produits des polynômes orthogonaux d'une variable peuvent être appliqués.

Dans le point $d$ ) de l'algorithme 2 on cherche une solution du problème linéaire des moindres carrés avec la condition additionnelle de non-négativité (NNLS - non negative least squares).

La procédure de résolution numérique de ce problème se trouve dans [2]. L'existence de $M(d)$ pour $\varepsilon_{d}$ donné sera démontrée plus loin. Il faut remarquer ici que le plus souvent $M(d)>N(d)$, tandis que la procédure présentée dans [2] s'applique sans aucune réserve au cas où le nombre des colonnes de la matrice ne dépasse pas celui de ses lignes. On peut, quand même, ajouter la condition de minimalisation de la longueur du vecteur $\left[B_{1}^{d}, B_{2}^{d}, \ldots, B_{M(d)}^{d}\right]$.

On voit facilement que cette modification ajoute de son côté $M(d)$ lignes nouvelles à la matrice du problème $d$ ). La nouvelle matrice est évidemment du rang $M(d)$, donc la procédure de [2] s'y applique sans aucun doute. 
THÉORÈME 3.2 : Étant donné $\varepsilon_{d}$, on peut toujours trouver $M(d)$ d'une telle manière que la condition (3.6) soit satisfaite.

Démonstration : Soit $Q_{j}^{d}, j=1,2, \ldots, M(d)$ un rectangle de la décomposition de $\Omega$ définie dans le point $b$ ) de l'algorithme 2 . Mettons

$$
B_{j}^{d}=\Delta_{Q_{j}^{d}} \phi_{x}, \quad j=1,2, \ldots, M(d) .
$$

Evidemment, $B_{j}^{d} \geqslant 0$, car la fonction $\phi_{x}$ est entièrement croissante. On voit que $\sum_{j=1}^{M(d)} \varphi_{k}\left(\mu_{j}^{d}\right) B_{j}^{d}, k=1,2, \ldots, N(d)$ sont les sommes de Rieman-Stieltjes de l'intégrale

$$
c_{k}=\int_{\Omega} \varphi_{k}(\lambda) d \phi_{x}(\lambda) \quad k=1,2, \ldots, N(d)
$$

Donc

$$
\sum_{k=1}^{N(d)}\left[\sum_{j=1}^{M(d)} \varphi_{k}\left(\mu_{j}^{d}\right)-c_{k}\right]^{2} \leqslant \varepsilon_{d}
$$

pourvu que $M(d)$ soit suffisamment grand. II

THÉORÈME $3.3: S i \phi_{x}(\lambda)=\|E(\lambda) x\|_{H}^{2}$ et $\phi_{x d}$ sont définies par l'algorithme 2 , alors la condition $(Q)$ est satisfaite. Cela implique (i) et (ii) du théorème 3.1.

Démonstration : Il suffit de prouver que la condition $(Q)$ est satisfaite. Soit $f \in C_{R}(\Omega)$. Pour $\varepsilon$ arbitraire mais tel que $0<\varepsilon<\frac{\|f\|_{0}}{\mathrm{\kappa}}$ choisissons un polynôme $Q_{m}$ du degré $\leqslant m$ tel que $\left\|f-Q_{m}\right\|_{\infty}<\varepsilon<\frac{\|f\|_{0}}{\kappa}$.

Nous avons $Q_{m}=\sum_{j=1}^{N(m)} \gamma_{j} \varphi_{j}$ pour $d$ assez grand. L'orthonormalité du système $\left\{\varphi_{j}\right\}$ implique $\gamma_{j}=\left(Q_{m}, \varphi_{j}\right)_{0}$ et $\sum_{j=1}^{N(m)} \gamma_{j}^{2}=\left\|Q_{m}\right\|_{0}^{2}$.

Donc

$$
\varepsilon \kappa \geqslant \kappa\left\|f-Q_{m}\right\|_{\infty} \geqslant\left\|f-Q_{m}\right\|_{0} \geqslant\left\|Q_{m}\right\|_{0}-\|f\|_{0}
$$

d'où il suit que

$$
\sum_{j=1}^{N(m)} \gamma_{j}^{2}=\left\|Q_{m}\right\|_{0}^{2} \leqslant\left[\kappa \varepsilon+\|f\|_{0}\right]^{2} \leqslant 4\|f\|_{0}^{2} .
$$


Comme $\varphi_{1}$ est du degré zéro $\left(\varphi_{1}=\right.$ Cte), donc $c_{1}=\varphi_{1} \int_{\Omega} d \phi_{x}(\lambda)$ et

$$
\begin{aligned}
\varphi_{1}^{2}\left[\int_{\Omega} d \phi_{x d}(\lambda)-\int_{\Omega} d \phi_{x}(\lambda)\right]^{2}=\varphi_{1}^{2}\left[\sum_{j=1}^{M(d)} B_{j}^{d}-\int_{\Omega} d \phi_{x}(\lambda)\right]^{2} \leqslant \\
\leqslant \sum_{k=1}^{N(d)}\left[\sum_{j=1}^{M(d)} \varphi_{k}\left(\mu_{j}^{d}\right) B_{j}^{d}-c_{k}\right]^{2} \leqslant \varepsilon_{d}
\end{aligned}
$$

d'où

$$
0 \leqslant \int_{\Omega} d \phi_{x d}(\lambda) \leqslant \int_{\Omega} d \phi_{x}(\lambda)+\frac{\sqrt{\varepsilon_{d}}}{\varphi_{1}} \leqslant 2 \int_{\Omega} d \phi_{x}(\lambda)=2\|x\|_{H}^{2}
$$

pour $d$ suffisamment grand. Choisissons maintenant $d \geqslant m$ et tel que les conditions (3.7), (3.8) et $\sqrt{\varepsilon_{d}}<\varepsilon$ soient satisfaites.

Nous avons

$$
\begin{aligned}
& \left|\int_{\Omega} f(\lambda) d \phi_{x}(\lambda)-\int_{\Omega} f(\lambda) d \phi_{x d}(\lambda)\right|=\mid \int_{\Omega}\left[f(\lambda)-Q_{m}(\lambda)\right] d \phi_{x}(\lambda)- \\
& \quad-\int_{\Omega}\left[f(\lambda)-Q_{m}(\lambda)\right] d \phi_{x d}(\lambda)+\int_{\Omega} Q_{m}(\lambda) d \phi_{x}(\lambda)-\int_{\Omega} Q_{m}(\lambda) d \phi_{x d}(\lambda) \mid \leqslant \\
& \quad \leqslant 3\left\|f-Q_{m}\right\|_{\infty}\|x\|_{H}^{2}+\left|\sum_{k=1}^{N(d)} \gamma_{k}\left[\sum_{j=1}^{M(d)} \varphi_{k}\left(\mu_{j}^{d}\right) B_{j}^{d}-c_{k}\right]\right| \\
& \quad \leqslant 3\|x\|_{H}^{2} \varepsilon+\left\{\sum_{k=1}^{N(d)} \gamma_{k}^{2} \sum_{k=1}^{N(d)}\left[\sum_{j=1}^{M(d)} \varphi_{k}\left(\mu_{j}^{d}\right) B_{j}^{d}-c_{k}\right]^{2}\right\}^{1 / 2}
\end{aligned}
$$

où on met par définition $\gamma_{k}=0$ pour tout $k$ tel que $N(m)<k \leqslant N(d)$.

Ainsi on arrive à la condition $(Q)$ :

$$
\left|\int_{\Omega} f(\lambda) d \phi_{x}(\lambda)-\int_{\Omega} f(\lambda) d \phi_{x d}(\lambda)\right| \leqslant 3\|x\|_{H}^{2} \varepsilon+2\|f\|_{0} \sqrt{\varepsilon_{d}} \leqslant K \varepsilon
$$

où $K$ ne dépend pas de $d$. III

Remarque : Nous avons déjà remarqué qu'en général $M(d)>N(d)$; donc l'algorithme 1 pourrait être considéré comme plus économique que l'algorithme 2. Observons que l'algorithme 1 nécessite la résolution d'un système des équations non linéaires, tandis que l'algorithme 2 est basé sur un classique problème algébrique de moindres carrés. 
Le théorème 3.4 suivant concerne l'ordre de convergence dans le cas spécial : $\lambda \in \rho(A)$. Cette information est bien sûr seulement partielle, mais semble être d'une certaine importance pratique. La convergence de l'algorithme dans $\rho(A)$ est la plus rapide (voir aussi [4]) et donc permet de nous informer relativement vite si le point qui nous intéresse est dans $\rho(A)$.

Dans [4] on trouve beaucoup plus d'informations sur l'ordre de convergence dans le cas où $A$ est autoadjoint.

Soit $\rho_{x}(A) \subset \mathbb{C}$ un tel ensemble que $\lambda \in \rho_{x}(A)$ si et seulement si il existe un rectangle normal $Q$ tel que $\lambda \in \operatorname{Int}(Q)$ et $\Delta_{Q} \phi_{x}=0$. On voit que $\rho_{x}(A)$ est ouvert et que $\rho(A) \subset \rho_{x}(A)$ (voir théorème 1.1).

Soit maintenant $Q$ un rectangle normal. Si $Q \subset \rho(A) \subset \rho_{x}(A)$, donc $\Delta_{Q} \phi_{x}=0$. On verra que $\Delta_{Q} \phi_{x d}$ satisfait cette relation approximativement et que $\Delta_{Q} \phi_{x d}$ converge à zéro très rapidement quand $d \rightarrow \infty$. L'application du théorème 3.4 qui suit nous permet d'approximer l'ensemble $\rho(A)$ en prenant la partie commune des approximations de $\rho_{x}(A)$ pour $x$ convenablement choisi et $d$ assez grand.

LEMME 3.4 (Élimination des faux éléments du spectre. Comparer [4]) : Soit $Q \subset \rho_{x}(A)$ un rectangle normal. Pour tout $p$ et $d$ naturels

$$
0 \leqslant \sum_{\mu_{j}^{d} \in Q} B_{j}^{d} \leqslant\left\{\begin{array}{cc}
\frac{C_{p}(s)}{s^{p}} & \text { pour l'algorithme 1, } \\
\left(1+C \sqrt{\varepsilon_{d}}\right) \frac{C_{p}(s)}{s^{p}}+C \sqrt{\varepsilon_{d}} & \text { pour l'algorithme 2, }
\end{array}\right.
$$

où $C$ est une constante, $C_{p}(s) \rightarrow 0$ quand $s \rightarrow \infty$ pour tout $p$ fixé, $\varepsilon_{d} \rightarrow 0$ quand $d \rightarrow \infty$ (voir la définition d'algorithme 2$), s=$ entier $(d / 2)$ et $B_{j}^{d} \geqslant 0, j=1,2, \ldots$ sont les coefficients définis par l'algorithme 1 ou 2.

Démonstration: Soit $Q^{\prime} \subset \rho_{x}(A)$ un rectangle normal tel que

$$
Q \subset \operatorname{Int}\left(Q^{\prime}\right) \subset \rho_{x}(A) .
$$

Il existe une fonction $f_{Q} \in C_{0}^{\infty}(\Omega)$ telle que $f_{Q}(\lambda) \geqslant 0$ pour tout $\lambda \in \Omega$, $f_{Q}(\lambda)=1$ pour $\lambda \in Q$ et $f_{Q}(\lambda)=0$ pour $\lambda \in \Omega \backslash Q^{\prime}$. Il est clair que

$$
\int_{\Omega} f_{Q}(\lambda) d \phi_{x}(\lambda)=0
$$

Mettons

$$
r(d)=\left\{\begin{array}{l}
N(d) \text { pour l'algorithme 1, } \\
M(d) \text { pour l'algorithme 2. }
\end{array}\right.
$$


On a

$$
\begin{aligned}
0 \leqslant \sum_{\mu_{j}^{d} \in Q} B_{j}^{d} \leqslant \sum_{j=1}^{r(d)} B_{j}^{d} f_{Q}\left(\mu_{j}^{d}\right)=\int_{\Omega} f_{Q}(\lambda) d \phi_{x d}(\lambda)= \\
=\int_{\Omega} f_{Q}(\lambda) d \phi_{x d}(\lambda)-\int_{\Omega} f_{Q}(\lambda) d \phi_{x}(\lambda) .
\end{aligned}
$$

Soit $W$ un polynôme arbitraire du degré $\leqslant d$. Donc

$$
\begin{aligned}
0 \leqslant \sum_{\mu_{j}^{d} \in Q} B_{j}^{d} & \leqslant \int_{\Omega}\left[f_{Q}(\lambda)-W(\lambda)\right] d \phi_{x d}(\lambda)- \\
& -\int_{\Omega}\left[f_{Q}(\lambda)-W(\lambda)\right] d \phi_{x}(\lambda)+\int_{\Omega} W(\lambda) d \phi_{x d}(\lambda)-\int_{\Omega} W(\lambda) d \phi_{x}(\lambda) .
\end{aligned}
$$

Comme (la condition $(Q)$ ) $\int_{\Omega} d \phi_{x d}(\lambda) \rightarrow \int_{\Omega} d \phi_{x}(\lambda)$ quand $d \rightarrow \infty$, la suite $\left\{\int_{\Omega} d \phi_{x d}(\lambda)\right\}$ est bornée et on peut écrire

$$
0 \leqslant \sum_{\mu_{j}^{d} \in Q} B_{j}^{d} \leqslant\left\|f_{Q}-W\right\|_{\infty} K+\left|\int_{\Omega} W(\lambda) d \phi_{x d}(\lambda)-\int_{\Omega} W(\lambda) d \phi_{x}(\lambda)\right|
$$

où $K$ est une constante.

Observons que pour l'algorithme $1 \int_{\Omega} W(\lambda) d \phi_{x d}(\lambda)=\int_{\Omega} W(\lambda) d \phi_{x}(\lambda)$.

La méthode déjà appliquée dans la démonstration du théorème 3.3 nous amène à l'inégalité suivante :

$$
\left|\int_{\Omega} W(\lambda) d \phi_{x d}(\lambda)-\int_{\Omega} W(\lambda) d \phi_{x}(\lambda)\right| \leqslant\|W\|_{0} \sqrt{\varepsilon_{d}}
$$

dans le cas d'algorithme 2. Comme $\|W\|_{0} \leqslant \kappa\left\|W-f_{Q}\right\|_{\infty}+\kappa\left\|f_{Q}\right\|_{\infty}$, nous arrivons finalement à la conclusion que

$$
0 \leqslant \sum_{\mu_{j}^{d} \in Q} B_{j}^{d} \leqslant \begin{cases}\left\|f_{Q}-W\right\|_{\infty} \cdot K & \text { pour l'algorithme 1, } \\ \left\|f_{Q}-W\right\|_{\infty}\left(K+\kappa \sqrt{\varepsilon_{d}}\right)+\kappa \sqrt{\varepsilon_{d}} & \text { pour l'algorithme 2. }\end{cases}
$$


Il nous suffit seulement de majoriser $\left\|f_{Q}-W\right\|_{\infty}$. Choisissons d'abord la fonction $f_{Q}$ d'une manière convenable. Soit

$$
Q=[\alpha, \beta] \times[\gamma, \delta] \text { et } Q^{\prime}=\left[\alpha^{\prime}, \beta^{\prime}\right] \times\left[\gamma^{\prime}, \delta^{\prime}\right],
$$

$Q \subset \operatorname{Int}\left(Q^{\prime}\right) \subset Q^{\prime} \subset \Omega$ et mettons $f_{Q}(u, v)=g_{1}(u) . g_{2}(v)$ où $g_{1} \in C_{0}^{\infty}\left(a_{1}, a_{2}\right)$, $g_{2} \in C_{0}^{\infty}\left(b_{1}, b_{2}\right), 0 \leqslant g_{1}(u) \leqslant 1,0 \leqslant g_{2}(v) \leqslant 1$ et

$$
\begin{aligned}
& g_{1}(u)=\left\{\begin{array}{lll}
1 & \text { pour } & u \in[\alpha, \beta] \\
0 & \text { pour } & u \in\left[a_{1}, a_{2}\right] \backslash\left[\alpha^{\prime}, \beta^{\prime}\right]
\end{array}\right. \\
& g_{2}(v)=\left\{\begin{array}{lll}
1 & \text { pour } & v \in[\gamma, \delta] \\
0 & \text { pour } & v \in\left[b_{1}, b_{2}\right] \backslash\left[\gamma^{\prime}, \delta^{\prime}\right] .
\end{array}\right.
\end{aligned}
$$

Soit $W(u, v)=W_{1}(u) W_{2}(v)$ où $W_{1}$ et $W_{2}$ sont les polynômes optimaux d'une variable, du degré $\leqslant s=$ entier $(d / 2)$, choisis pour les fonctions $g_{1}$ et $g_{2}$ respectivement. On a

$$
\begin{aligned}
&\left\|f_{Q}-W\right\|_{\infty} \leqslant\left\|g_{1}-W_{1}\right\|_{\infty}+\left\|g_{1}-W_{1}\right\|_{\infty}\left\|g_{2}-W_{2}\right\|_{\infty}+ \\
&+\left\|g_{2}-W_{2}\right\|_{\infty}
\end{aligned}
$$

car $\left\|g_{1}\right\|_{\infty}=\left\|g_{2}\right\|_{\infty}=1$. Appliquons maintenant le théorème de Jackson [7] ( $W_{1}$ et $W_{2}$ sont optimaux !) :

$$
\begin{aligned}
& \left\|g_{1}-W_{1}\right\|_{\infty} \leqslant K_{p} \omega_{1 p}(s) \frac{\left(a_{2}-a_{1}\right)^{p}}{s^{p}} \\
& \left\|g_{2}-W_{2}\right\|_{\infty} \leqslant K_{p} \omega_{2 p}(s) \frac{\left(b_{2}-b_{1}\right)^{p}}{s^{p}} .
\end{aligned}
$$

Ici $p$ est naturel quelconque, $K_{p}$ est une constante qui dépend de $p$, mais ne dépend pas de $s ; \omega_{1 p}$ et $\omega_{2 p}$ sont quelques modules de continuité, donc $\omega_{i p}(s) \rightarrow 0, i=1,2$ quand $s \rightarrow \infty$. Cela termine la démonstration. III

THÉORÈME 3.4 : Soit $Q \subset \rho_{x}(A)$ un rectangle normal. Si $\phi_{x d}$ est définie par l'algorithme 1 ou 2, alors

$$
0 \leqslant \Delta_{Q} \phi_{x d} \leqslant\left\{\begin{array}{cc}
\frac{C_{p}(s)}{s^{p}} & \text { pour l'algorithme 1 } \\
\left(1+C \sqrt{\varepsilon_{d}}\right) \frac{C_{p}(s)}{s^{p}}+C \sqrt{\varepsilon_{d}} & \text { pour l'algorithme 2 }
\end{array}\right.
$$

où $p, C, C_{p}$ et s sont comme dans le lemme 3.4.

R.A.I.R.O. Analyse numérique/Numerical Analysis 
Démonstration : Pour $\lambda$ fixé mettons

$$
K(\lambda)=\{\mu \in \mathbb{C} \mid[\operatorname{Re}(\mu) \leqslant \operatorname{Re}(\lambda)] \text { et }[\operatorname{Im}(\mu) \leqslant \operatorname{Im}(\lambda)]\} .
$$

Observons que

$$
\phi_{x d}(\lambda)=\sum_{j=1}^{r(d)} B_{j}^{d} H\left(\lambda, \mu_{j}^{d}\right)=\sum_{\mu j \in K(\lambda)} B_{j}^{d}
$$

$(r(d)$ - comme dans la démonstration du lemme 3.4), donc

$$
\Delta_{Q} \phi_{x d}=\sum_{\mu_{j}^{d} \in Q \backslash L B(Q)} B_{j}^{d} \leqslant \sum_{\mu_{j}^{d} \in Q} B_{j}^{d} .
$$

L'application du lemme 3.4 termine la démonstration. $\quad \|$

\section{RÉFÉRENCES}

1. A. K. Azız, Mathematical foundations of the finite element method. New York, 1972.

2. C. L. Lawson, R. J. Hanson, Solving least squares problems. Prentice-Hall, 1974.

3. S. ŁoJASIEWICZ, Wstep do teorii funkcji rzeczywistych. PWN Warszawa, 1973.

4. K. MoszyŃsKI, On approximation of the spectral density function of a selfadjoint operator. To appear in Studia Scientiarum Mathematicarum Hungarica, № 14, 1979.

5. K. MoszYŃsKi, Approximation of the spectrum of a bounded, normal operator with the help of its spectral density functions. Preprint № 249. Institute of Mathematics, Polish Academy of Sciences, Warsaw, oct. 1981.

6. Sz. F. Riesz, B. NAGY, Leçons d'analyse fonctionnelle. Akademiai Kiado. Budapest, 1952.

7. T. J. Rivin, An introduction to the approximation of functions. Blaisdell Publ., 1969.

8. A. SARD, Linear approximation. AMS 1963.

9. A. H. STroud, Approximate calculation of multiple integrals. Prentice-Hall, 1971. 